\title{
Evaluation of the Effects of Freezing on the Preservation of Fresh Shiitake Fruit Bodies and Lenthionine Formation after Thawing
} \author{
OMURA $^{3}$ \\ ${ }^{1}$ Department of Biological Resource Sciences, Miyazaki University, Miyazaki 889-21, Japan \\ ${ }^{2}$ Miyazaki JA Food Research and Development Inc., Miyazaki 880, Japan \\ ${ }^{3}$ Department of Food Science and Technology, Kyushu University, Fukuoka 812-81, Japan
}

Masahito Suiko, ${ }^{1}$ Kazuo NishiYama, ${ }^{1}$ P.H. Prasantha Fernando, ${ }^{1}$ Koichi Sugita, ${ }^{2}$ Michio MiUra ${ }^{1}$ and Hirohisa

Received September 13, 1996

\begin{abstract}
The effects of freezing-thawing on the physical and chemical properties of shiitake were evaluated. It was observed that freezing-thawing did not cause apparent changes in the physical state of shiitake, although a characteristic sulfurous odor was generated. We demonstrated that lenthionine was produced during the thawing of frozen shiitake, and the amount produced varied with the conditions of both freezing and thawing. Among the conditions tested, freezing at $-10^{\circ} \mathrm{C}$ and thawing at $37^{\circ} \mathrm{C}$ were found to be the most suitable for the production of lenthionine.
\end{abstract}

Keywords: shiitake, lenthionine, freezing, thawing

Shiitake (Lentinus edodes) is a major edible mushroom in Asia, and it has long been consumed as a relished food item in Japan and in the neighboring countries. Shiitake is known to have a very special characteristic taste and aroma, and this unique character is attributed to two chemical compounds, viz., guanosine-5'-monophosphate (5'-GMP), and 1,2,3,5, 6-pentathiepane (lenthionine). $5^{\prime}$-GMP is produced by the ribonuclease-catalyzed hydrolysis of RNA; whereas lenthionine is synthesized from its precursor by damage to the fresh fruit bodies or during the rehydration of dried shiitake. Along with inosine- $5^{\prime}$-monophosphate, $5^{\prime}$-GMP is widely used as a seasoning ingredient.

The characteristic odor of shiitake is generated when dried shiitake is soaked in lukewarm water, or when the fresh fruit body is chopped into small pieces and allowed to stand for a while. The chemical agent possessing this flavor is lenthionine, which was first identified by Morita and Kobayashi $(1966,1967)$. Its physicochemical characteristics and application to food were reported by Wada et al. (1967a, b). Furthermore, the enzymatic system involved in the synthesis of lenthionine was reported by Iwami (1977). During the process of the formation of lenthionine, lentinic acid (precisely, its desglutamyl derivative) gives rise to thiosulfinate and formaldehyde which both possess antifungal activities. Incidentally, lenthionine has also been shown to be an antimicrobial agent with a broad spectrum of antifungal activity (Morita \& Kobayashi, 1967), which may play an important role during the growth of shiitake.

In this paper, we describe the effects of different freezing and thawing conditions on the quality preservation of shiitake fruit bodies with respect to changes in its physicochemical properties including touch, color and odor, as well as lenthionine formation.

\section{Materials and Methods}

Raw shiitake (Yakult Haru-Aki No. 2) mushrooms were harvested in the University Forests of Miyazaki University in March, 1993. In addition, some shiitake mushrooms were purchased at a local market in Miyazaki, Japan. Crystalline lenthionine from the Takeda Chemical Industries, Ltd., Osaka, was used as a standard for determination by gas liquid chromatography (GLC).

Sample preparation

Samples $A$ and $B$ Raw shiitake ( $200 \mathrm{~g}$ ) was frozen at $-20^{\circ} \mathrm{C}$ and 2 days later was ground into small pieces at 18,000 rpm for 3 min using a homogenizer (Nihon Seiki HED-2 blender). The homogenized shiitake was mixed with distilled water $(200 \mathrm{~g})$ and divided into two portions. One portion was kept at room temperature and the other at $37^{\circ} \mathrm{C}$. After a $3.5 \mathrm{~h}$ incubation, the homogenates were filtered through first a nylon mesh cloth and then a Whatman No. 1 filter paper.

Samples $C$ and $D$ Raw shiitake $(200 \mathrm{~g})$ was frozen at $-10^{\circ} \mathrm{C}$ and 2 days later was ground into small pieces as described above. Filtration of the homogenates were carried out as described for samples A and B.

Sample $E$ Raw shiitake $(100 \mathrm{~g})$ was frozen at $-10^{\circ} \mathrm{C}$ and 2 days later was kept at room temperature for $18 \mathrm{~h}$ and treated as mentioned above.

Sample $F$ Dried shiitake (100 g) was soaked in water $(100 \mathrm{~g})$ at room temperature. Twenty-two hours later, the extract was collected and concentrated by freeze-drying.

Quantification of lenthionine by gas chromatography The shiitake extract $(20 \mathrm{ml})$ was placed in test tubes with caps, to which $\mathrm{NaCl}(1 \mathrm{~g})$ and purified $n$-hexane $(2 \mathrm{ml})$ were added with stirring for $5 \mathrm{~min}$. After allowing the samples to stand for a while, the hexane layer was collected with a Pasteur pipette, and the hexane droplets which were suspended in the aqueous 
Table 1. Quality evaluation and quantification of lenthionine from raw shiitake (Lentinus edodes) by freezing and thawing.

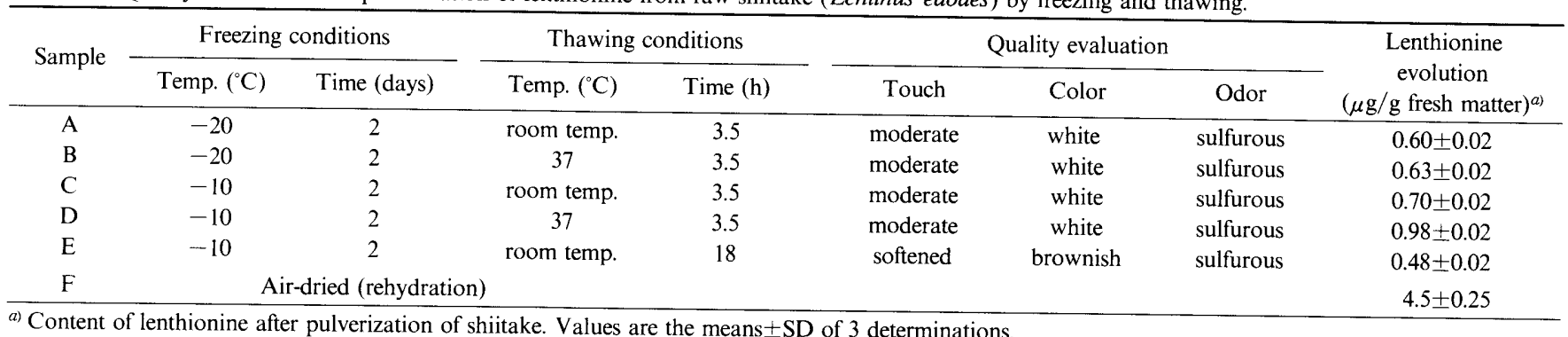

a) Content of lenthionine after pulverization of shiitake. Values are the means \pm SD of 3 determinations.

phase were separated by centrifugation at $10,000 \mathrm{rpm}$ for 15 min, collected, and combined with the hexane layer first obtained. A Shimadzu GC-7A gas chromatograph equipped with a flame photometric detector (FPD) was used for the quantification of high sulfur-containing lenthionine as described previously (Ito et al., 1978). The retention times of hexane and lenthionine were $0.7 \mathrm{~min}$ and $18.3 \mathrm{~min}$, respectively.

\section{Results and Discussion}

Rehydrated dry shiitake has a characteristic flavor which is attributable to lenthionine. Unlike 5'-GMP, however, lenthionine is not present in intact shiitake but is produced through enzymatic action from its precursor(s) during postharvest processing. Therefore, in the post-harvest handling of shiitake, especially in commercial production, it is important to adopt methods that would give rise to the effective production of lenthionine.

The results of the quality evaluation of shiitake after freezing-thawing are shown in Table 1 . In the case of samples A-D, the touch, color and odor were moderate (slightly softer compared to a fresh sample), white, and sulfurous, except that sample E was softened, brownish and sulfurous. These physicochemical properties varied slightly depending on the moisture content of the shiitake.

Quantitative analysis of lenthionine was performed using GLC after pulverizing and thawing each sample. The concentrations of lenthionine in the 6 extracts (samples A-F) are summarized in Table 1. It seems likely that the amount of lenthionine produced in each sample is somewhat higher under the progressing conditions of enzymatic reaction than that present in a mature state of the shiitake. As described in Materials and Methods, all extracts were obtained from $100 \mathrm{~g}$ of either raw (Samples A-E) or dried (Sample F) shiitake. However, it should be considered that the average moisture content of raw shiitake is around $90 \%$, whereas in dried shiitake it is not beyond $10 \%$. Among the samples A-E, the content of lenthionine was found to be the highest for sample $\mathrm{D}$, indicating that, with respect to the freezing and thawing conditions, freezing at $-10^{\circ} \mathrm{C}$ and thawing at $37^{\circ} \mathrm{C}$ were the preferable conditions for higher lenthionine production. Furthermore, pulverization prior to thawing (sample D) is the best for the highest lenthionine production. Lenthionine has been shown to be produced from its precursor, lentinic acid, by mutual reaction of gamma-glutamyl transferase and cysteine sulfoxide lyase, during post-harvest treatment (Iwami, 1977). The enzymatic action takes place during the thawing process and is more facilitated by thawing at $37^{\circ} \mathrm{C}$ than at room temperature. Within the disrupted tissue, the two enzymes and their respective substrates can easily come into contact, as in the case of sample D. Because sample D has the highest lenthionine content, these freezing and thawing conditions may be useful in making large amounts for seasoning production.

In the case of dried shiitake (sample F), hydration (addition of water) was used for production of lenthionine (Table 1). Comparison of the lenthionine concentrations in samples $\mathrm{D}$ and $\mathrm{F}$ reveals that the freezing-thawing method was either as good as or even better than the dry preservationrehydration method. This confirms that freezing at $-10^{\circ} \mathrm{C}$ is an effective method for the preservation of shiitake, because this procedure can produce the characteristic flavor of this mushroom, although the natural texture and freshness of shiitake change slightly on thawing.

Finally, it should be noted that although dehydration is commonly used for long-term preservation of shiitake, we have tested the effects of freezing and found virtually no significant changes in the appearance even after shiitake had been kept frozen (at $-20^{\circ} \mathrm{C}$ ) for more than 2 years. Moreover, we observed a strong sulfurous smell during the boiling process; therefore, a considerable amount of lenthionine might have vaporized. However, the shiitake still contained lenthionine after boiling. When the sample was pre-treated by freezing-thawing, the amount of lenthionine extracted increased to approximately twice that of its untreated counterpart (data not shown). People have been using shiitake and its characteristic flavor-imparting ingredient, lenthionine, in a wide variety of food preparation, especially together with meat and fish since ancient times. The lipids in foods may prevent the evaporation of lenthionine during cooking procedures by effectively absorbing it. Lenthionine may also mask the undesirable odor of some cooking substances. It is especially interesting to consider that since ancient times people have selected dry preservation and rehydration based on experience and have found the optimum suitable conditions for lenthionine production. Although $\mathrm{pH} 7-8$ was the desirable $\mathrm{pH}$ range for lenthionine production, $\mathrm{pH} 4$ is the optimum $\mathrm{pH}$ to prevent lenthionine degradation. However, at $\mathrm{pH} 4$, no lenthionine production was observed (data not shown). This study confirms that, in addition to the traditional method of shiitake presevation, freezing could also be used in keeping with modern trends in food processing. 


\section{References}

Ito, Y., Toyoda, M., Suzuki, H. and Iwaida, M. (1978). Gas-liquid chromatographic determination of lenthionine in shiitake mushroom (Lentinus edodes) with special reference to the relation between carbon disulfide and lenthionine. J. Food Sci, 43, 12871289.

Iwami, K. (1977). Enzymatic development of flavor in Lentinus edodes. Nippon Nógeikagaku Kaishi, 68, R39-R46 (in Japanese).

Morita, K. and Kobayashi, S. (1966). Isolation and synthesis of lenthionine, an odorous substance of shiitake, an edible mushroom.
Tetrahedron Lett., 1966, 573-577.

Morita, K. and Kobayashi, S. (1967). Structure, and synthesis of lenthionine and its analogs. Chem. Pharm. Bull., 15, 988-993.

Wada, S., Nakatani, H., Fujinawa, S., Kimura, H. and Hagaya, M. (1967a). Studies on lenthionine, a new aroma-bearing substance from shiitake (part 1). Eiyo to Shokuryo, 20, 355-359 (in Japanese). Wada, S., Nakatani, H., Toda, J. and Hagaya, M. (1967b). Studies on lenthionine, a new aroma-bearing substance from shiitake (part 2). Eiyo to Shokuryo, 20, 360-362 (in Japanese). 